\title{
MOLECULAR BEAM EPITAXY OF II-VI BASED HETEROSTRUCTURES
}

\author{
LESLIE A. KoLodzIEJSKI, \\ Department of Electrical Engineering and Computer Science, \\ Massachusetts Institute of Technology, \\ Cambridge, MA 02139, USA
}

ROBERT L. GUNSHOR, School of Electrical Engineering,Purdue University, West Lafayette, IN 47907, USA

ARTo V. NURMIKKo

Division of Engineering and Department of Physics, Brown University, Providence, RI 02912, USA

\author{
AND NoBuo OtsuKa \\ Materials Engineering,Purdue University, \\ West Lafayette, IN 47907, USA
}

(Received August 8, 1990)

The nonequilibrium growth technique of molecular beam epitaxy (MBE) has provided for the fabrication and investigation of a multitude of novel layered heterostructures based on II-VI compound semiconductors. The ability to grow epitaxial metastable magnetic and semimagnetic semiconductors layered with conventional II-VI semiconductors has resulted in structures which, for example, exhibit frustrated antiferromagnetism, and a wide wavelength tunability due to selftrapping of excitons in ZnTe-containing layered structures and due to extremely large $(\approx 1 \mathrm{eV})$ quantum shifts of light emission from $\mathrm{MnTe} / \mathrm{CdTe}$ superlattice structures. In addition, the control in the stoichiometry of surfaces and the composition of molecular beams used in the MBE growth technique has allowed for the fabrication of very advanced heterostructures which have combined the II-VI and III-V families of compound semiconductors. The work which will be described in the following review represents a very small sampling of the many important results achieved in the field of II-VI based heterostructures. The topics have been selected to illustrate and provide an example of the utility of $\mathrm{MBE}$ and the potential of "engineered" II-VI heterostructures and quantum wells. 
PACS numbers; 68.55.Bd;78.65Fa

\section{Introduction}

The II-VI family of compound semiconductors has long been recognized for potential optical and optoelectronic device applications due to the presence of their wide, direct energy bandgaps. Figure 1 represents a composite of many of

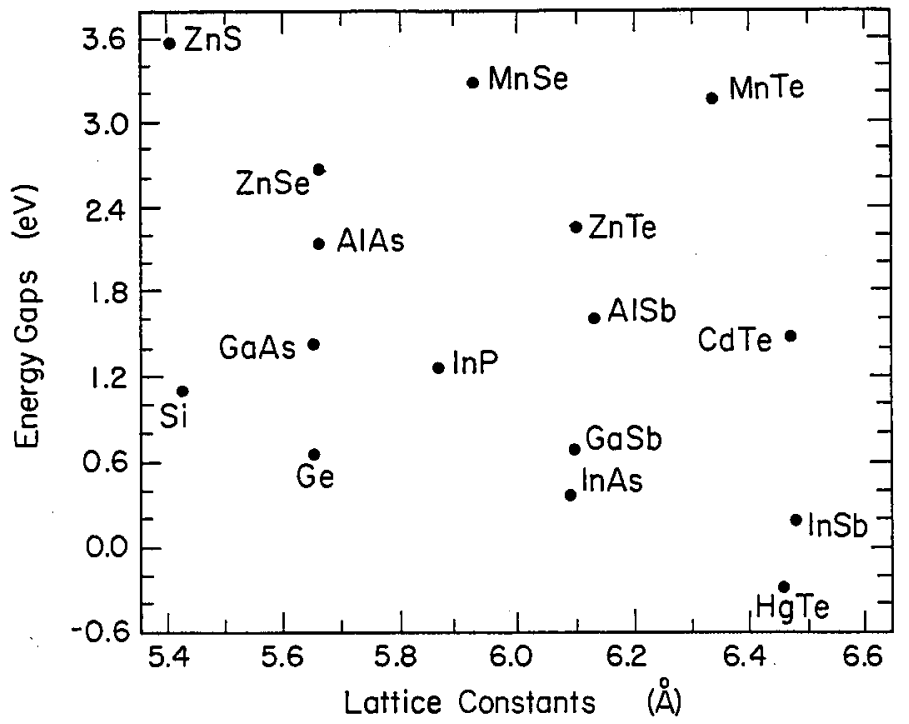

Fig. 1. Comparison of the room temperature energy bandgap and lattice parameter for many of the technologically important III-V, II-V, and elemental semiconductors can be easily seen.

the technologically important compound and elemental semiconductors and compares their energy bandgaps and lattice parameters. Immediately apparent from Fig. 1 is the wide wavelength range (from 3.66 to $-0.30 \mathrm{eV}$ ) which is spanned by the II-VI materials. In contrast, III-V semiconductors span a much smaller range of wavelengths and may exhibit indirect energy bandgaps, similar to the elemental semiconductors $\mathrm{Si}$ and $\mathrm{Ge}$. The addition of $\mathrm{Mn}$ to various II-VI compounds results in a substantial increase in the energy bandgap, paralleling the effect that alloying aluminum has on binary III-V materials. Although the lattice parameter is also seen to vary, Mn-containing semimagnetic semiconductors have been utilized in a variety of strained-layer configurations making possible investigations of the optical, microstructural, and electronic properties of II-VI heterostructures and multiple quantum wells. In addition, by employing the nonequilibrium growth of 
molecular beam epitaxy, metastable, unstrained, thick epitaxial layers of $\mathrm{MnSe}$ and $\mathrm{MnTe}$ in zincblende crystalline structure have been fabricated. In the case of $\mathrm{MnSe}$, the equilibrium bulk structure is $\mathrm{NaCl}$, whereas bulk $\mathrm{MnTe}$ exhibits the NiAs crystal structure. Each of the aforementioned magnetic semiconductors in its zincblende form has been layered with narrower gap II-VI semiconductors and has thus formed the barrier or wider bandgap layer in quantum well configurations. By careful selection of the substrate upon which the layered structure is epitaxially deposited, the strain can be distributed throughout or may be confined to a particular layer of the superlattice. In most cases, the various II-VI based heterostructures and quantum wells have been grown on III-V substrates, primarily due to their superior crystalline quality and ease of surface preparation for epitaxy. II-VI bulk substrates are still relatively scarce in large area and high quality.

As can be seen in Fig. 1, for almost any combination of II-VI materials a closely lattice matched III-V compound is available for use a substrate. Conversely, for most of the technologically important III-V semiconductors, a closely lattice-matched wide bandgap II-VI material is available for use as a II-VI $p s e u$ doinsulator. The similarities in lattice parameters, crystal structures, and thermal expansion coefficients, but the differences in bandgaps, suggesting substantial band discontinuities in layered configurations, strongly suggests investigation of advanced II-VI/II-V heterostructures. The II-VI layer provides i) a means to passivate the surface of the III-V semiconductor in the absence of a native oxide or ii) as a replacement for deposited amorphous insulators or for wider bandgap epitaxial III-V materials which may lack sufficient band discontinuities in both the conduction and valence bands. Substantial progress has been realized in the field of II-VI based heterostructures and multiple quantum wells in the last five years primarily through the successful growth of the II-VIs by molecular beam epitaxy and metalorganic vapor phase epitaxy. The following review addresses only work based on the MBE growth technique, and cannot possibly cover all of the successes achieved by the many investigators working worldwide in the field. Therefore, selected topics will be discussed which, in the authors' opinion, illustrate various interesting physical phenomena which were observed in unique and novel II-VI based heterostructures and multiple quantum wells. The objective is to stimulate creativity in efforts to overcome the remaining crystal growth problems of the II-VI family of semiconductors by engineering new layered structures and by continuing pursuit of advanced nonequilibrium crystal growth techniques.

\section{ZnSe superlattice structures containing zincblende MnSe}

$\mathrm{ZnSe}$ has been layered with the II-VI semiconductor compounds $\mathrm{ZnS}, \mathrm{ZnTe}$, $\mathrm{Zn}(\mathrm{S}, \mathrm{Se}),(\mathrm{Zn}, \mathrm{Cd}) \mathrm{Se}$, the semimagnetic semiconductor $(\mathrm{Zn}, \mathrm{Mn}) \mathrm{Se}$, and the magnetic semiconductor MnSe to form wide bandgap II-VI superlattices and multiple quantum well (MQW) structures. For all of these materials the lattice constant varies substantially from that of $\mathrm{ZnSe}$, such that layered structures form strained-layer superlattices, provided the layer thicknesses are below the critical thickness where misfit dislocations form. The following section addresses superlattice structures composed of $\mathrm{ZnSe}$ and $\mathrm{MnSe}$; some recent references reviewing 
the properties of other ZnSe-based superlattices and MQWs can be found in [1-4]. The diluted magnetic semiconductor $(\mathrm{Zn}, \mathrm{Mn}) \mathrm{Se}$ is formed by alloying the magnetic transition metal $\mathrm{Mn}$ into the II-VI compound $\mathrm{ZnSe}$. The Mn atoms randomly replace $\mathrm{Zn}$ atoms modifying the band structure through an increase in the direct energy bandgap. The two $s$ electrons of the outer shell of the Mn atoms replace those of $\mathrm{Zn}$ and become part of the band electrons in extended states. The five electrons in the unfilled $3 d$ shell of $\mathrm{Mn}$, however, give rise to localized magnetic moments which are partially aligned in an external magnetic field. The resultant magnetic moment interacts with the band electrons causing a Zeeman splitting which is two orders of magnitude larger at low lattice temperature than for the host II-VI semiconductor. The presence of the magnetic ion (with the associated Zeeman shifts) provides an unique and useful feature to the superlattices and MQWs in which Mn is incorporated, as we shall see below. The very interesting magnetic and magneto-optical properties of a wide variety of diluted or semimagnetic semiconductors are described by Gaj et al. [5] and by Aggarwal et al. [2].

Although the presence of the magnetic transition metal Mn results in very interesting magnetic and magneto-optical properties, the primary motivation for the thin film growth of $(\mathrm{Zn}, \mathrm{Mn})$ Se originated in the necessity of obtaining a wider bandgap material than $\mathrm{ZnSe}$, such that layered quantum microstructures based on $\mathrm{ZnSe}$ could be fabricated. In epitaxial layers of ( $\mathrm{Zn}, \mathrm{Mn}) \mathrm{Se}$, as the $\mathrm{Mn}$ fraction was increased, the direct energy bandgap was found to increase; however, the increase was found to be rather small for a substantial increase in Mn fraction [4]. To attempt to provide sufficient band offset for significant confinement of both electrons and holes in the quantum well structures, substantial $\mathrm{Mn}$ fractions would be necessary for fabrication of the wider bandgap or barrier layers which would be alternated with the $\mathrm{ZnSe}$ well layers. The main difficulty which became apparent was the tendency of bulk crystals of $(\mathrm{Zn}, \mathrm{Mn}) \mathrm{Se}$ to exhibit mixed crystalline phases and hexagonal crystal structure when greater than approximately $25 \% \mathrm{Mn}$ was incorporated into the host zincblende lattice [6]. By employing the nonequilibrium growth technique of molecular beam epitaxy (MBE), zincblende metastable epitaxial layers of $(\mathrm{Zn}, \mathrm{Mn}) \mathrm{Se}$ were deposited over the entire composition range onto $\mathrm{ZnSe}$ buffer layers grown on GaAs substrates $[7,8]$. Due to the significant lattice mismatch, relatively thick epitaxial layers were strain relieved by the generation of misfit dislocations. From data of X-ray diffraction measurements, the lattice parameter of thick layers of zincblende $(\mathrm{Zn}, \mathrm{Mn}) \mathrm{Se}$ is seen to vary substantially with Mn fraction [4]. Utilizing the flexibility of MBE and the ability to modulate the layer composition on the scale of a monolayer, MQWs were fabricated at relatively low substrate temperatures $\left(\sim 400^{\circ} \mathrm{C}\right)$ using elemental sources of $\mathrm{Zn}, \mathrm{Se}$ and vacuum distilled $\mathrm{Mn}$. Various microstructures, composed of differing amounts of $\mathrm{Mn}$ in the barrier layers and $\mathrm{ZnSe}$ well layers, were grown having significant amounts of strain due to the differing in-plane lattice parameters between layers; strained-layer superlattices existed whereby the strain was elastically accommodated throughout the very thin layers without the generation of dislocations. The resultant $(\mathrm{Zn}, \mathrm{Mn})$ Se MQWs have been characterized by a variety of techniques including transmission electron microscopy, photoluminescence and photoluminescence excitation spectroscopy, time resolved luminescence spectroscopy, nonlinear 
absorption spectroscopy, and observations of stimulated emission and gain spectra; these measurements have been reviewed by Kolodziejski et al. [9, 4].

Photoluminescence measurements of epitaxial layers of $(\mathrm{Zn}, \mathrm{Mn}) \mathrm{Se}$ at low temperatures exhibited the presence of two competing and dominant optical transitions which originated from the radiative recombination of excitons near the bandedge, as well as due to emission from transitions associated with an excited Mn-ion. For small Mn fractions $(0<x<0.1)$, blue excitonic recombination dominates, whereas very intense yellow luminescence due to the Mn-ion internal transitions dominates at higher $\mathrm{Mn}$ fractions. For samples containing Mn fractions greater than 0.5 , the only emission observed is the yellow emission around $2.1 \mathrm{eV}[10]$. As can be seen in the Fig. 2b, an epitaxial film of ( $\mathrm{Zn}, \mathrm{Mn})$ Se, having $33 \% \mathrm{Mn}$, exhibits a photoluminescence spectrum containing significant amounts of yellow luminescence; whereas very little luminescence associated with recombination of excitons near the bandedge is observed. In striking contrast to this spectrum however, is the photoluminescence spectrum (Fig. 2a) emitted from a superlattice containing barriers of $\mathrm{Zn}_{0.67} \mathrm{Mn}_{0.33} \mathrm{Se}$ and $\mathrm{ZnSe}$ wells. Although the $\mathrm{MQW}$ sample is illuminated with above bandgap radiation, creating electron and hole pairs in the $(\mathrm{Zn}, \mathrm{Mn}) \mathrm{Se}$ barrier layers, the carriers are quickly and efficiently swept to the narrow bandgap ZnSe well where the carriers radiatively recombine emitting strongly intense blue light representative of the effective bandgap of the quantum-sized structure. In comparison to the yellow luminescence emitted from the Mn-ion internal transition, the blue emission due to the bandedge transition is approximately 100 times more intense.

The ability to incorporate increasing fractions of $\mathrm{Mn}$ into the $\mathrm{ZnSe}$ host lattice, while maintaining the zincblende crystal structure, motivated further pursuit of the MBE growth of a metastable zincblende form of MnSe. For the growth of both thick epitaxial layers and $\mathrm{MnSe} / \mathrm{ZnSe}$ superlattice structures, only vacuum distilled $\mathrm{Mn}$ and elemental Se was used for the growth which occurred at $400^{\circ} \mathrm{C}$. By extrapolating the data of energy bandgap and lattice parameter versus $\mathrm{Mn}$ fraction for the zincblende epilayers of the alloy, the values of $3.4 \mathrm{eV}$ and $5.93 \AA$, respectively, were obtained for zincblende MnSe. Thus when MnSe was layered with $\mathrm{ZnSe}$, the MnSe served as the barrier material; due to a lattice mismatch of $4.7 \%$, the superlattices were strained-layer structures. The resolution of monolayer growth offered by molecular beam epitaxy also suggested that the magnetic properties of ultra thin magnetic semiconductors could be. investigated by controlling the spacing of, and spacing between, Mn-containing layers. To achieve the objective of controlling the growth to a monolayer, reflection high energy electron diffraction (RHEED) intensity oscillations were employed during the growth. One period of oscillation in the intensity of the specular spot of the diffraction pattern indicated that only one monolayer of $\mathrm{MnSe}$ or $\mathrm{ZnSe}$ was deposited. A series of "comb-like" superlattices, consisting of 30 to 100 periods, were grown with $\mathrm{MnSe}$ layer thicknesses of one, three, and four monolayers; the MnSe layer thicknesses were separated by $45 \AA$ of $\mathrm{ZnSe}$. For purposes of comparison, structures were also grown having one and three monolayers of $\mathrm{MnSe}$ where the growth was interrupted after each $\mathrm{MnSe} / \mathrm{ZnSe}$ interface, in an effort to fabricate interfaces with minimum growth front roughness. An additional structure was fabricated consisting of ten 

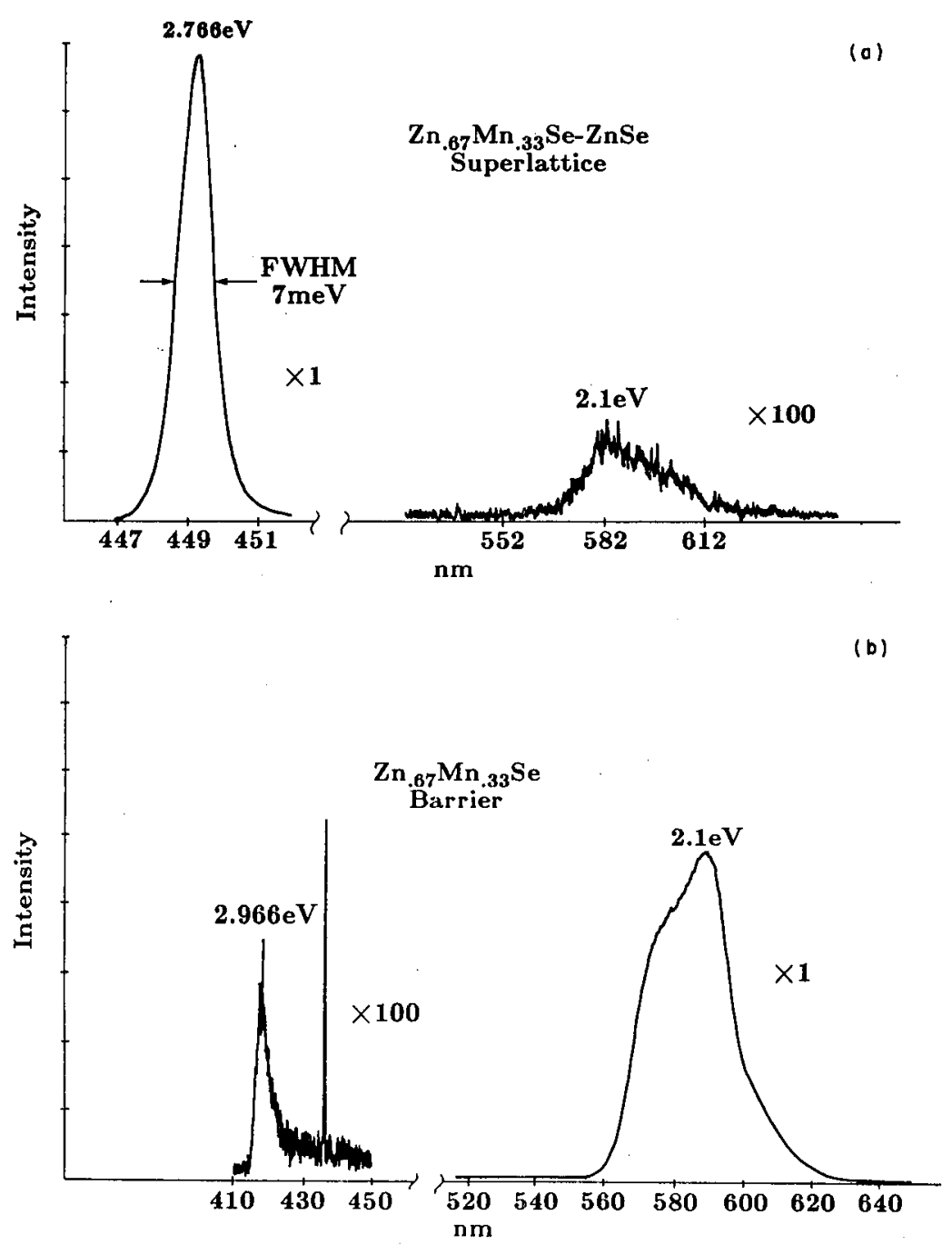

Fig. 2. A comparison of the photoluminescence intensity versus wavelength from (a) a $(\mathrm{Zn}, \mathrm{Mn}) \mathrm{Se} / \mathrm{ZnSe}$ multiple quantum well structure having $\mathrm{ZnSe}$ wells and $(\mathrm{Zn}, \mathrm{Mn}) \mathrm{Se}$ barriers containing $33 \% \mathrm{Mn}$ and (b) a thick epitaxial layer of ( $\mathrm{Zn}, \mathrm{Mn})$ Se containing $33 \%$ $\mathrm{Mn}$. The temperature of the measurement was $6.5 \mathrm{~K}$ and both samples were illuminated with ultraviolet radiation under similar conditions. 
monolayers of MnSe separated by $24 \AA$ of $\mathrm{ZnSe}$ with a total of 30 periods (to minimize the amount of strain in the structure).

Photoluminescence measurements were performed in the presence of a magnetic field (up to $5 \mathrm{~T}$ ), and were used as an early indicator of the magnetic properties of the $\mathrm{MnSe} / \mathrm{ZnSe}$ superlattices; complementary information was provided through direct magnetization measurements obtained using a SQUID magnetometer. In the absence of a magnetic field the photoluminescence peak due to the ground state excitonic transition occurred at an energy that was expected from Kronig-Penney models used for superlattices containing $\mathrm{ZnSe}$ with ultrathin layers of $\mathrm{MnSe}$ barriers $\left(E_{g}=3.4 \mathrm{eV}\right)$. However in the presence of a magnetic field, the ground state optical transitions were red-shifted due to the exchange interaction between electron-hole states of the superlattice and the magnetic moments of the $\mathrm{Mn}$ ions in the MnSe layers. The shift of the optical transitions as a function of applied field is shown in Fig. 3. Data is plotted for both Faraday and Voigt configurations of the applied magnetic field. As the MnSe layer thickness was reduced, significant and unexpected Zeeman shifts were observed and suggested a significant amount of paramagnetic behavior. However, bulk crystals of rock-salt MnSe exhibited antiferromagnetic behavior and bulk crystals of $(\mathrm{Zn}, \mathrm{Mn}) \mathrm{Se}$ existing in the wurtzite phase showed an increasing tendency of antiferromagnetic ordering as the Mn mole fraction was increased. In addition, MBE grown epilayers of ( $\mathrm{Zn}, \mathrm{Mn}) \mathrm{Se}$ showed a reduction in Zeeman shift as the Mn fraction increased suggesting an increasing antiferromagnetic coupling between $\mathrm{Mn}$-ion spins.

Complementary magnetization measurements of the $\mathrm{MnSe} / \mathrm{ZnSe}$ superlattice structures, obtained with a SQUID magnetometer, confirmed the presence of a positive contribution at low temperature for the samples with ultrathin layers of $\mathrm{MnSe}$, while the thick epitaxial layer of $\mathrm{MnSe}$ exhibited typical antiferromagnetic ordering behaviour [11]. Although further studies are required to appreciate fully the origin of the frustrated antiferromagnetism, the tendency for spins to align in the presence of an external magnetic field is believed to arise from "loose" spins existing at the heterointerfaces between $\mathrm{MnSe}$ and ZnSe. For very thin layers of $\mathrm{MnSe}$, the heterointerfaces will dominate, whereas for thicker $\mathrm{MnSe}$ layers the "bulk" of the layer will dominate the magnetic behavior. The presence of the Mn atoms provides a magnetic probe on the scale of chemical bond lengths due to the short range superexchange interaction between nearest neighbor Mn-ions coupled through the Se anions. Incomplete layer growth, which is intrinsic to the MBE growth process, may occur even though the layers have grown in a layer-by-layer manner. These interfacial islands will have a finite size and may be effective in frustrating the antiferromagnetic ordering which is expected for MnSe. In addition, reconstruction effects may play a role during the MBE growth in the presence of a significant degree of interface strain. Further studies of the behaviour of the $\mathrm{MnSe} / \mathrm{ZnSe}$ superlattices in the presence of a magnetic field may provide additional information for superlattice structures in general when considering issues relevant to heterointerfaces formation. 


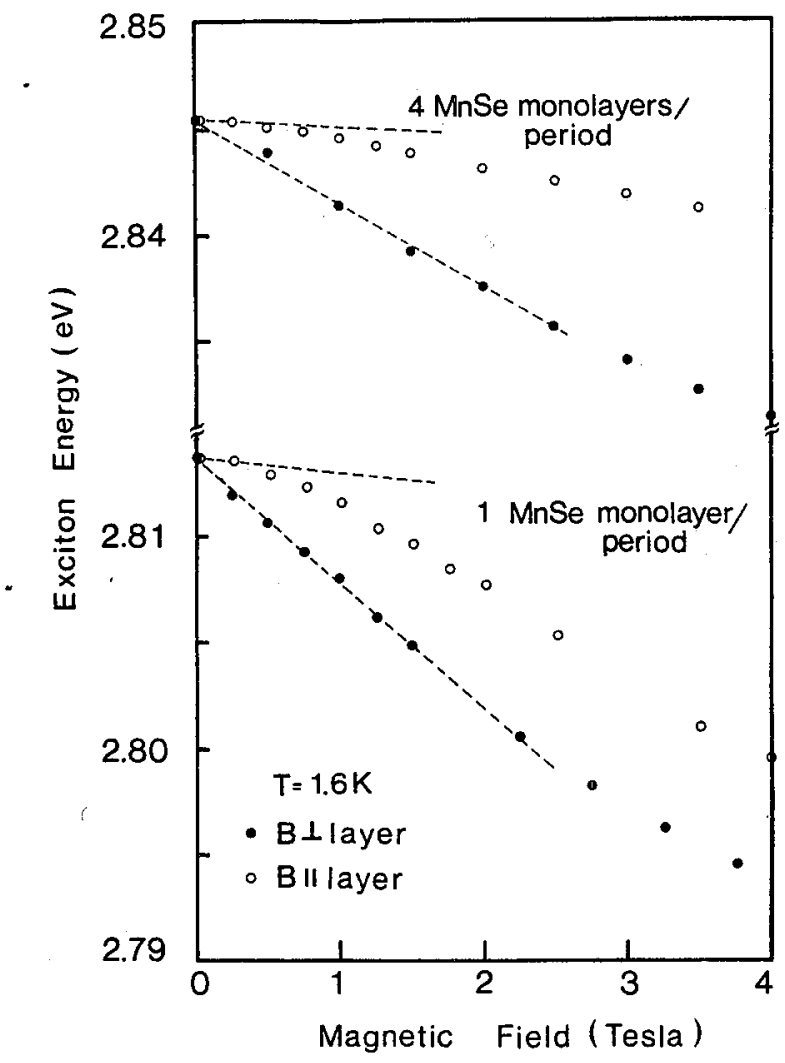

Fig. 3. Energy of ground state transition observed in photoluminescence as a function of applied magnetic field for $\mathrm{MnSe} / \mathrm{ZnSe}$ superlattice structures containing one, four, and ten monolayers of MnSe. Significant shift for one and four monolayer MnSe structures indicates presence of observed paramagnetic behavior.

\section{Ultrathin Sheets of ZnTe within (Zn,Mn)Se/ZnSe Multiple Quantum Wells}

Magneto-optical measurements of the many types of quantum-sized structures, composed of $(\mathrm{Zn}, \mathrm{Mn}) \mathrm{Se}$ and $\mathrm{ZnSe}$, suggested that the band discontinuity between conduction and valence bands resided primarily in the conduction band. Measurements of the energy bandgap by photoluminescence and reflectance of the $(\mathrm{Zn}, \mathrm{Mn})$ Se epilayers indicated the bandgap differences between well and barrier layers of the MQW structures ranged between $100-300 \mathrm{meV}$. By carefully studying the magnetic field induced shifts of optical transitions, which was associated with the exchange interaction of the hole wavefunction residing in the $(\mathrm{Zn}, \mathrm{Mn}) \mathrm{Se}$ barrier layers, Hefetz et al. [10] determined that the valence band offset was very small $(\sim 20 \mathrm{meV})$ and may be dependent on the strain present in a particu- 
lar $(\mathrm{Zn}, \mathrm{Mn}) \mathrm{Se} / \mathrm{ZnSe} \mathrm{MQW}$ sample. The rapid decrease in the photoluminescence quantum efficiency as the temperature increased suggested that the excitons nonradiatively recombined due to lack of confinement of the hole in the very shallow quantum well. The objective to obtain large quantum efficiencies at room temperature suggested the need to provide additional confinement of the hole, while maintaining the confinement of the electron, to encourage radiative recombination of excitons in the MQW structures. By comparing the electron affinities of many II-VI compounds, ZnTe possessed a valence band nearer to the vacuum level than either $(\mathrm{Zn}, \mathrm{Mn}) \mathrm{Se}$ or $\mathrm{ZnSe}$; if $\mathrm{Zn}(\mathrm{Se}, \mathrm{Te})$ was used as the well material, would the hole be confined in $(\mathrm{Zn}, \mathrm{Mn}) \mathrm{Se} / \mathrm{Zn}(\mathrm{Se}, \mathrm{Te}) \mathrm{MQW}$ structures?

During the initial experiments associated with the MBE growth of the mixed crystal alloy of $\mathrm{Zn}(\mathrm{Se}, \mathrm{Te})$, an extreme difficulty was encountered when a small fraction of Te was desired. In the work reported by Yao et al. [12], over the entire range of Te fraction, a Te-to-Se flux ratio of three to ten was required. Using the high vapor pressure elements for sources during MBE, extreme control of the oven temperature is required and very difficult to achieve. To circumvent the problems associated with controlling the alloy concentrations, ultrathin layers of $\mathrm{ZnTe}$ were embedded within ( $\mathrm{Zn}, \mathrm{Mn}) \mathrm{Se} / \mathrm{ZnSe} \mathrm{MQW}$ structures. The ultrathin layers of $\mathrm{ZnTe}$ were spaced appropriately such that an approximate mixed crystal of $\mathrm{Zn}(\mathrm{Se}, \mathrm{Te})$ with small Te fraction was formed; in such a case a $\mathrm{Zn}(\mathrm{Se}, \mathrm{Te})$ "pseudo-alloy" was conveniently deposited by MBE. Figure 4 illustrates the effect of placing an ultrathin sheet ( $3 \AA$ or one monolayer) of $\mathrm{ZnTe}$ in the center of $\mathrm{ZnSe}$ wells existing in a $(\mathrm{Zn}, \mathrm{Mn}) \mathrm{Se} / \mathrm{ZnSe} \mathrm{MQW}$ structure. During the growth of the $\mathrm{ZnTe}$ layer, atomic layer epitaxy growth techniques were employed on recovered surfaces to minimize the interface roughness of the $\mathrm{ZnSe} / \mathrm{ZnTe}$ heterointerface.

In Figure 4a, typical photoluminescence intensity versus energy is measured for a $\mathrm{Zn}_{0.77} \mathrm{Mn}_{0.23} \mathrm{Se} / \mathrm{ZnSe} \mathrm{MQW}$ structure containing no ultrathin sheets of $\mathrm{ZnTe}$, and is similar to the spectrum recorded in Fig. 2 (a). The only luminescence originates from very intense blue emission of ground state excitonic optical transitions. In striking contrast is the spectrum recorded in Fig. $4 \mathrm{~b}$ for the same MQW structure, but now containing one ultrathin $(3 \AA)$ sheet of $\mathrm{ZnTe}$ placed in the center of each $44 \AA \mathrm{ZnSe}$ well. The broad luminescence features are at much lower energies and are due to the strong localization of excitons at the $\mathrm{ZnSe} / \mathrm{ZnTe}$ heterointerfaces. The photoluminescence features are similar to that measured for bulk mixed crystals of $\mathrm{Zn}(\mathrm{Se}, \mathrm{Te})$ with low fractions of $\mathrm{Te}$ [13]. Figure $4 \mathrm{c}$ indicates that the lowest energy exciton transition (in absorption) has not been significantly shifted by the presence of the ultrathin $\mathrm{ZnTe}$ sheets. In addition to the luminescence occurring at a range of wavelengths, the radiative recombination efficiency was greatly enhanced as the temperature was increased from LHe temperatures.

Comparison of the temperature dependent optical properties of the bulk mixed crystals with the optical properties of these "engineered" MQWs, with and without application of a magnetic field, has provided for a detailed understanding of the exciton self-trapping phenomena associated with the presence of $\mathrm{Te}$ at the $\mathrm{ZnSe} / \mathrm{ZnTe}$ heterointerface [14]. As mentioned above, the magnitude of the Zeeman shift can be directly related to the extent of the hole wavefunction into the $(\mathrm{Zn}, \mathrm{Mn}) \mathrm{Se}$ barrier layers. It was determined in the MQWs containing the 


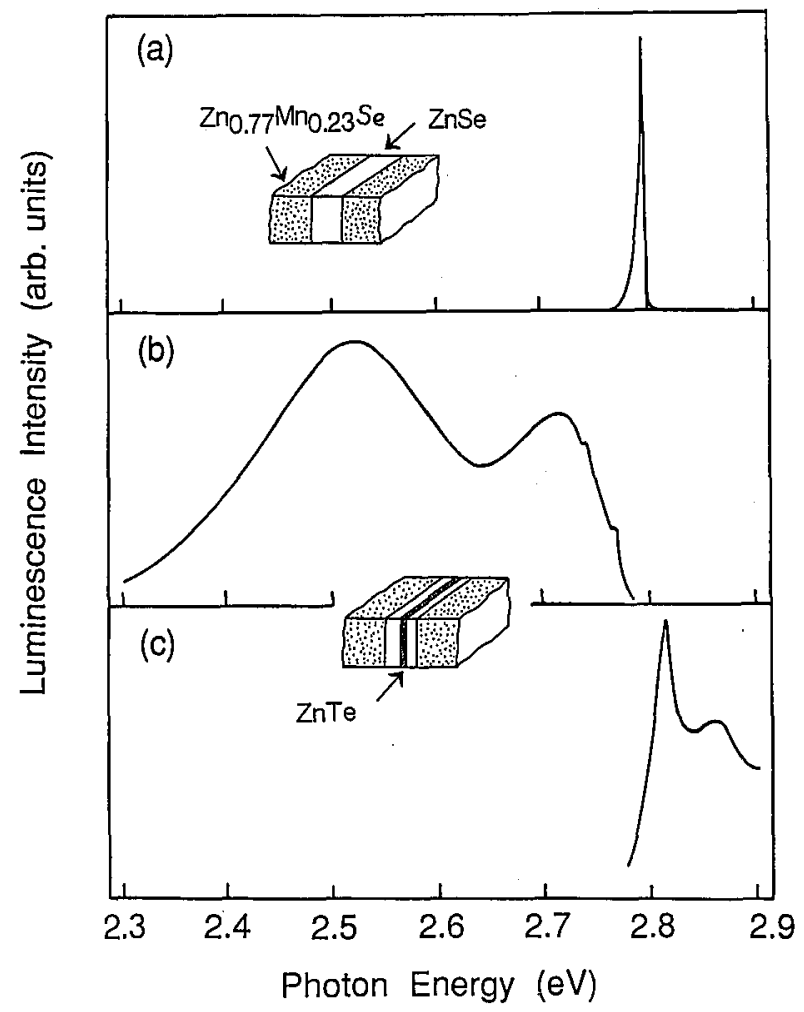

Fig. 4. Comparison of photoluminescence intensity versus wavelength for a $(\mathrm{Zn}, \mathrm{Mn}) \mathrm{Se} / \mathrm{ZnSe}$ multiple quantum well: (a) without the presence of an ultrathin sheet of $\mathrm{ZnTe}$, (b) with the presence of an ultrathin ( $3 \AA$ ) sheet of $\mathrm{ZnTe}$ place in the center of the $\mathrm{ZnS}$ well, and (c) photoluminescence excitation spectrum of multiple quantum well in (b) containing the ultrathin $\mathrm{ZnTe}$ sheet. The measurement was performed under. identical conditions of UV illumination and at $8 \mathrm{~K}$.

ultrathin $\mathrm{ZnTe}$ sheets that the hole component of the exciton wavefunction becomes strongly localized at Te-centers, reducing the exciton's effective Bohr orbit. The two features observed in Fig. $4 \mathrm{~b}$ are identified to excitons localized to isolated single and double Te sites, which are expected to be located at the $\mathrm{ZnSe} / \mathrm{ZnTe}$ heterointerface. The high radiative efficiency and the relatively long lifetimes which have been measured for the MQW structures, further suggests that the hole is strongly localized with the electron in a Coulomb orbit. Originally motivated to confine the hole within the $\mathrm{Zn}(\mathrm{Se}, \mathrm{Te})$ well of a $(\mathrm{Zn}, \mathrm{Mn}) \mathrm{Se} / \mathrm{Zn}(\mathrm{Se}, \mathrm{Te}) \mathrm{MQW}$, the hole is very strongly localized due to the presence of Te as an isoelectronic impurity. As the ZnTe layer thickness is increased, a two-dimensional isoelectronic impurity "band" can be formed and investigations with regard to the formation of band offsets can begin to be developed. In addition, for device applications such 
localization is highly desirable if occurring at room temperature to prohibit the nonradiative recombination of excitons freely moving throughout the crystal.

\section{Zincblende MnTe/CdTe Superlattices}

As noted above, the presence of ultrathin sheets of $\mathrm{ZnTe}$ embedded within engineered $(\mathrm{Zn}, \mathrm{Mn}) \mathrm{Se} / \mathrm{ZnSe} \mathrm{MQW}$ structures provided for the emission of light over a very large wavelength range. Blue $(\sim 2.6 \mathrm{eV})$ light has also recently been observed from the ve ry large quantum-shifted emission from superlattices composed of $\mathrm{MnTe} / \mathrm{CdTe}[15,16]$. In these structures the well material is $\mathrm{CdTe}$ $\left(E_{g}=1.593 \mathrm{eV}\right)$, whereas the barrier material is zincblende MnTe. The nonequilibrium crystal growth technique of MBE allowed for the fabrication of the metastable phase of zincblende MnTe; the equilibrium crystal structure is NiAs with an energy bandgap of $1.3 \mathrm{eV}$. Extrapolating the data of bandgap and lattice parameter for alloys of bulk crystals of zincblende $(\mathrm{Cd}, \mathrm{Mn}) \mathrm{Te}$, suggests values of $3.18 \mathrm{eV}$ $[17]$ and $6.34 \AA[18]$, respectively. X-ray diffraction measurements on thin films of zincblende MnTe provide lattice parameter values of $6.33 \AA$, and optical reflectance indicates that the bandgap is approximately $3.20 \mathrm{eV}$ in close agreement with the extrapolated values. Both X-ray diffraction powder camera measurements. and electron diffraction via RHEED and transmission electron microscopy (TEM) have confirmed the zincblende crystal structure of the metastable MnTe layers.

By employing the zincblende MnTe as barrier layers, single quantum well (SQW) structures have been fabricated with CdTe well thicknesses varying from 56 to $10 \AA$. The SQWs were fabricated on CdTe buffer layers which were deposited onto closely lattice-matched MBE-grown InSb homoepitaxial buffer layers. Due to the lattice mismatch between CdTe and MnTe, the thickness of MnTe layers remained constant at $35 \AA$ in the SQW structures; TEM indicated the MnTe layers were pseudomorphic and free of misfit dislocations. Figure 5 illustrates the large quantum shifts observed in photoluminescence as the CdTe well thickness decreased. The peaks observed in photoluminescence originate from optical transitions from the $n=1$ conduction band to the $n=1$ valence band states. For the narrowest $(10 \AA) \mathrm{CdTe}$ wells studied thus far, the luminescence at low temperature is in the blue portion of the spectrum and represents a quantum shift due confinement of the carriers of $1 \mathrm{eV}[15,16]$.

\section{II-VI/III-V Heterostructures}

Thus far this review has emphasized the MBE growth of II-VI/II-VI quantum well structures which have in all cases been fabricated onto III-V bulk substrates. Utilization of the III-V substrate is largely due to the superior crystalline quality, reasonably large areas, and relatively modest cost. In Figure 1 the degree of lattice mismatch between the aforementioned material combinations can be determined. However, there are many examples of II-VI/III-V heterostructure combinations for which the degree of lattice mismatch is very small or the layered configuration is perfectly lattice matched. In addition, all of the III-V and many 


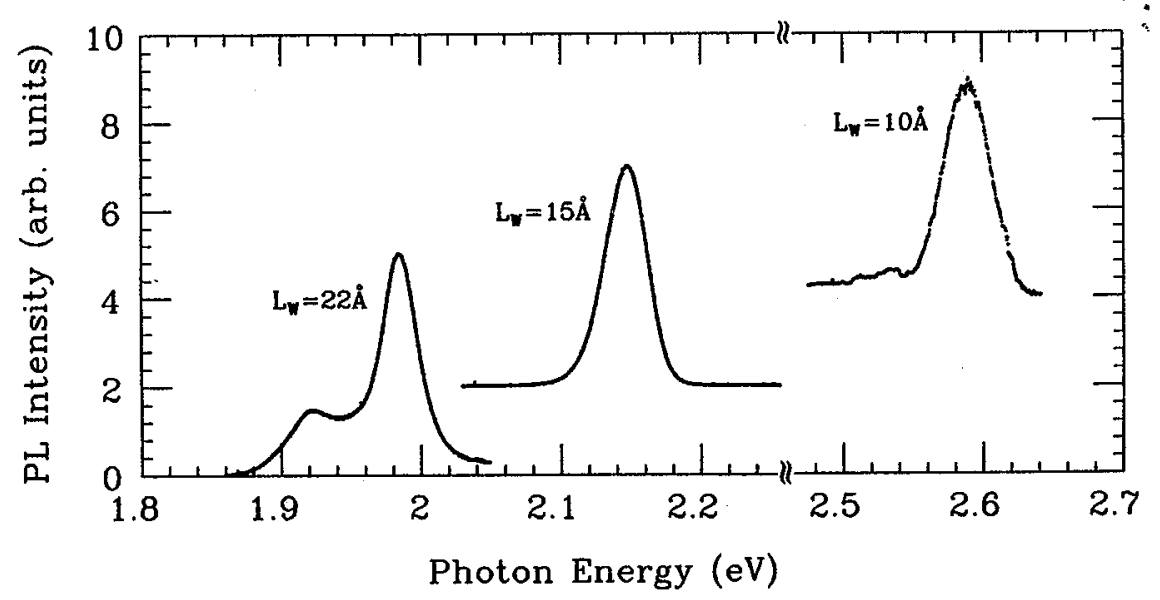

Fig. 5. Low temperature $(5 \mathrm{~K})$ photoluminescence intensity versus wa velength for various $\mathrm{MnTe} / \mathrm{CdTe}$ superlattice structures having differing well dimensions as shown in the figure.

of the II-VI semiconductors are of zincblende crystal structure, and have thermal expansion coefficients which do not vary dramatically from one compound to another, in contrast to thermal expansion coefficients for insulators such as $\mathrm{SiO}_{2}$. The substantially larger energy bandgaps of the II-VI family of semiconductors suggest that substantial band discontinuities might exist (in many cases) when a II-VI material is layered with a III-V material. With the complementary nature of the II-VI and III-V compound evident, II-VI/III-V heterostructures and multiple quantum wells grown by MBE are currently under investigation. The objective is to utilize epitaxial II-VI materials as pseudoinsulators on epitaxial layers of III-V semiconductors. The II-VI material may provide the wide bandgap barrier layer, as in the case of $\mathrm{CdTe} / \mathrm{InSb}$ multiple quantum wells, or may provide a passivation layer such as in $\mathrm{ZnSe} / \mathrm{GaAs}$ heterostructures.

The development of a metal-insulator-semiconductor (MIS) technology based on the important compound semiconductor GaAs has met with considerable difficulties due to the inadequate electrical characteristics of interfaces formed using native oxides or by deposition of various insulators onto the GaAs surface. In the "non-epitaxial" insulator/GaAs interfaces, the existence of a large number of interface states $[19,20,21]$ limits the extent to which the bands can be bent. An alternative technology has been developed, however, in which the wider bandgap semiconductor $(\mathrm{Al}, \mathrm{Ga}) \mathrm{As}$ is used as a semi-insulator in a variety of field effect transistor structures. An epitaxial heterojunction is utilized in these transistor structures due to the nearly identical lattice match between (Al,Ga)As and $\mathrm{GaAs}$, and due to the ability to fabricate such a heterointerface without growth interruption at the electrical junction. A very different epitaxial II-VI/III-V heterojunction has recently been employed in a field effect transistor structure where pseudomor- 
phic $\mathrm{ZnSe}$ [22] forms a pseudoinsulator on doped epitaxial layers of GaAs. Typical transistor characteristics were exhibited, and channel modulation indicated that the Fermi level could be varied over a large portion of the GaAs bandgap [23]. The wider bandgap of $\mathrm{ZnSe}$, compared to that of $(\mathrm{Al}, \mathrm{Ga}) \mathrm{As}(2.0 \mathrm{eV}$ for an $\mathrm{Al}$ mole fraction of 0.5 ), suggests a variety of device applications where $\mathrm{ZnSe}$ may present an alternative to $(\mathrm{Al}, \mathrm{Ga}) \mathrm{As}$ for passivation of $\mathrm{GaAs}$.

A series of metal-insulator-semiconductor capacitors have been fabricated for which the capacitance-voltage $(C-V)$ characteristics have been investigated $[24,25]$. Following the growth of the $\mathrm{ZnSe} / p$-GaAs heterojunctions, capacitors (125 $\mu \mathrm{m}$ in diameter) were formed using a lift-off for the Al metal gate. Many samples have been studied and consist of both $n$ - and $p$-type MBE-grown GaAs, where the GaAs has been grown in a completely separate III-V dedicated growth chamber. Following the "typical" growth of the $\mathrm{ZnSe} / \mathrm{GaAs}$ structure, the capacitors have been fabricated in the as-grown condition or have been treated with a post deposition anneal; subsequent structures have been fabricated wherein the interfaces exhibit various GaAs surface reconstructions in RHEED patterns prior to nucleation of $\mathrm{ZnSe}$. The $C-V$ measurements illustrate that the structure can be biased into accumulation ( $p$-type GaAs), depletion, inversion ( $n$-type GaAs), and deep depletion. A substantial band offset therefore exists in the valence band, but a relatively small conduction band offset is expected from these data. Using Terman's [26] method to calculate interface state densities, the interface state densities have been obtained as a function of the GaAs bandgap, and were compared with that reported by Chung et al. [27] for the (Al,Ga)As/GaAs interface in Fig. 6 . The particular structure for which the $C-V$ data was used, is for a capacitor which experienced a post deposition anneal, but is representative of the data obtained in as-grown structures which were grown with a Ga-rich surface of GaAs prior to nucleation of the insulator, $\mathrm{ZnSe}$. As can be seen in the figure, the interface state density can be very low and comparable to that observed in a more typical III-V/III-V heterointerface.

In the case of $\mathrm{ZnSe} / \mathrm{GaAs}$ heterostructures, the $\mathrm{ZnSe}$ is used as an epitaxial pseudoinsulator to passivate the GaAs surface. In the case of $\mathrm{CdTe} / \mathrm{InSb}$ multiple quantum well structures however, the CdTe represents a wide energy bandgap barrier layer whereas InSb is the well material. The two materials are very closely lattice matched $(<0.05 \%)$, and both theoretical predictions [28] and experimental measurements [29] indicate the quantum wells are of Type I with substantial conduction and valence band discontinuities. Large quantum shifts in the bandgap energy are predicted for the relatively wide quantum wells as a result of the small effective mass of the electrons and light holes. For example, a $75 \AA$ quantum well has a ground state transition energy twice that of bulk InSb [30]. Structures involving reasonable well dimensions allow a wavelength range of $2-5.5 \mu \mathrm{m}$ to be accessed. Figure 7 shows a cross-sectional TEM micrograph of a CdTe/InSb $\mathrm{MQW}$ structure grown by MBE in a single growth chamber [31,32]. The dark contrast represents the $163 \AA$ InSb well, while the light contrast is the $167 \AA \mathrm{CdTe}$ barrier layer; in the structure there are 20 periods to form the MQW. In the growth of the MQW structures of CdTe/InSb, the InSb is grown at very low temperatures $\left(280^{\circ} \mathrm{C}\right)$ with an $\mathrm{Sb}$ craker to provide a source of $\mathrm{Sb}_{2}$ dimers. Although 


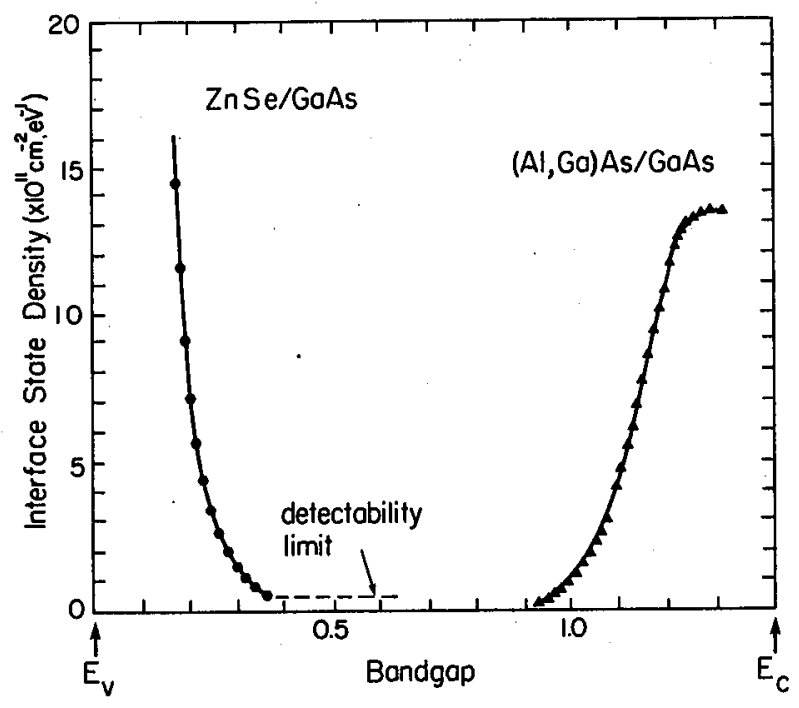

Fig. 6. Interface state density as a function of the GaAs bandgap for a metal-insulator-semiconductor capacitor structure composed of an epitaxial $\mathrm{ZnSe}$ insulator on an epitaxial $\mathrm{GaAs}$ surface. Also shown is the reported interface state density for an $(\mathrm{Al}, \mathrm{Ga}) \mathrm{As} / \mathrm{GaAs}$ interface [27].

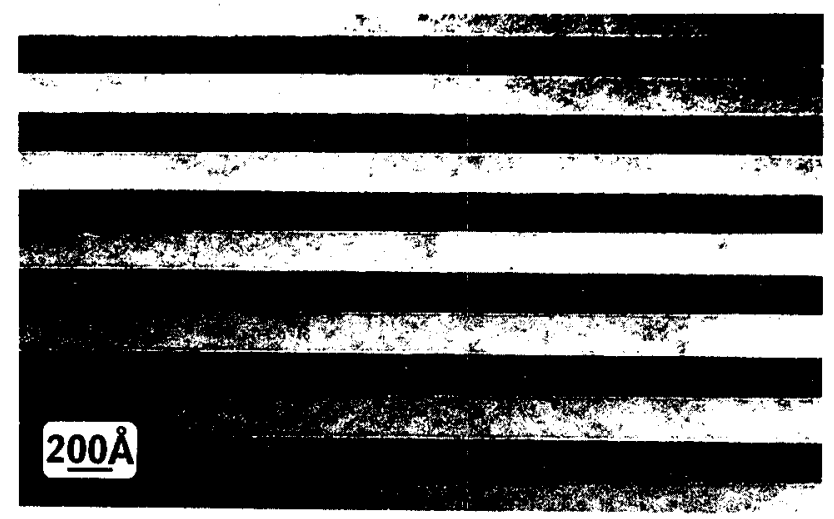

Fig. 7. Cross-sectional transmission electron microscope image obtained from a $\mathrm{CdTe} / \mathrm{InSb}$ multiple quantum well structure. The structure contains 20 periods of 163 $\AA$ InSb and $167 \AA$ of CdTe grown on an MBE-grown InSb homoepitaxial buffer layer. 
much investigation is still required to fully understand the electronic, optical and microstructural properties of these novel II-VI/III-V MQWs, the initial results show promise for their fabrication. The rapid success provides further motivation to pursue additional II-VI/III-V heterostructure combinations, in addition to utilizing newer growth techniques for their fabrication such as chemical beam epitaxy.

\section{Summary}

The objective of the review of II-VI based heterostructures was to emphasize the differing manners in which bandgap engineering, in addition to use of the nonequilibrium MBE growth techniques, provided novel structures which exhibited interesting physical phenomena with potential for device applications. II-VI MQWs of sophisticated structure have exhibited a wide wavelength range of optical emission due to i) the presence of exciton self trapping in $(\mathrm{Zn}, \mathrm{Mn}) \mathrm{Se} / \mathrm{ZnSe}$ containing ultrathin sheets of $\mathrm{ZnTe}$ and ii) large $(1 \mathrm{eV})$ quantum shifts from $\mathrm{MnTe} / \mathrm{CdTe}$ superlattices. The growth by molecular beam epitaxy of MnSe and MnTe has resulted in metastable zincblende crystal structure, and interesting magnetic phenomena such as frustrated antiferromagnetism in $\mathrm{MnSe} / \mathrm{ZnSe}$ superlattices. The future directions of II-VI materials may include expanding their use for integration in II-VI/III-V heterostructures where the II-VI is the pseudoinsulator or barrier layer for III-V based devices.

\section{Acknowledgement}

The authors would like to gratefully acknowledge the many graduate students, research engineers, and co-authors on the cited publications for their dedication and contributions to the research described above. The research on II-VI semiconductors was supported by Office of Naval Research, Air Force Office of Scientific Research, Defense Advanced Research Projects Agency, and the National Science Foundation.

\section{References}

[1] Yao, Taakafumi, The Technology and Physics of Molecular Beam Epitaxy, E.H.C. Parker, ed., Plenum Press, New York 1985, p. 313.

[2] R.L. Aggarwal, J.K. Furdyna, S. von Molnar, eds., Diluted Magnetic (Semimagnetic) Semiconductors, Proceedings of the Materials Research Society, Vol. 89, (1987).

[3] T.C. McGill, C.M. Sotomayor Torres, W. Gebhardt, eds., Growth and Optical Properties of Wide-Gap II-VI Low Dimensional Structures, NATO ASI Series B, Vol. 200, Plenum Press, New York 1988.

[4] L.A. Kolodziejski, R.L. Gunshor, A.V. Nurmikko, Crystal Properties and Preparation, R.M. Biefeld, ed., Vol. 21, Trans Tech Publications, Switzerland, 1989, p. 199. 
[5] J.A. Gaj, J. Ginter, R.R. Galazka, Phys. Status Solidi B 89, 655 (1978).

[6] A. Twardowski, T. Dietl, M. Demianuk, Solid State Commun. 64, 63 (1983).

[7] L.A. Kolodziejski, R.L. Gunshor, T.C. Bonsett, R. Venkatasubramanian, S. Datta, R.B. Bylsma, W.M. Becker, N. Otsuka, Appl. Phys. Lett. 47, 160 (1985).

[8] L.A. Kolodziejski, R.L. Gunshor, N. Otsuka, B.P. Gu, Y. Hefetz, A.V. Nurmikko, Appl. Phys. Lett. 47, 1482 (1986).

[9] L.A. Kolodziejski, R.L. Gunshor, N. Otsuka, S. Datta, W.M. Becker, A.V. Nurmikko, IEEE J. Quantum Electron. QE-22, 1666 (1986).

[10] Y. Hefetz, W.C. Goltsos, A.V. Nurmikko, L.A. Kolodziejski, R.L. Gunshor, S. Datta, Appl. Phys. Lett. 48, 372 (1986).

[11] S.-K. Chang, D. Lee, H. Nakata, A.V. Nurmikko, L.A. Kolodziejski, R.L. Gunshor, J. Appl. Phys. 62, 4835 (1987).

[12] T. Yao, Y. Makita, S. Maekawa, J. Cryst. Growth 45, 309 (1978).

[13] D. Lee, A. Mysyrowicz, A.V. Nurmikko, B.J. Fitzpatrick, Phys. Rev. Lett. 58, 1475 (1987).

[14] Q. Fu, D. Lee, A.V. Nurmikko, L.A. Kolodziejski, R.L. Gunshor, Phys. Rev. B 38, 3173 (1989).

[15] S.M. Durbin, J. Han, O. Sungki, M. Kobayashi, D.R. Menke, R.L. Gunshor, Q. Fu, N. Pelekanos, A.V. Nurmikko, D. Li, J. Gonsalves, N. Otsuka, Appl. Phys. Lett. 55, 2087 (1989).

[16] Q. Fu, J. Ding, W. Walecki, A.V. Nurmikko, S.M. Durbin, J. Han, M. Kobayashi, R.L. Gunshor Phys. Rev. B, in press. (1990).

[17] Y. Lee, A. Ramdas, Phys. Rev. B 38,10600 (1988).

[18] D.R. Yoder-Short, U. Debska, J.K. Furdyna, J. Appl. Phys. 58, 4056 (1985).

[19] H.H. Wieder, J. Vac. Sci. Technol. 15, 1498 (1978).

[20] L.G. Meiners, J. Vac. Sci. Technol. 15, 1402 (1978).

[21] E. Kohn, H.L. Hartnagel, Solid-State Electron. 21, 409 (1978).

[22] R.L. Gunshor, L.A. Kolodziejski, M.R. Melloch, M. Vaziri, C. Choi, N. Otsuka, Appl. Phys. Lett. 50, 200 (1987).

[23] G.D. Studtmann, R.L. Gunshor,L.A. Kolodziejski, M.R. Melloch , J.A. Cooper, R.F. Pierret, D.P. Munich, C. Choi, N. Otsuka, Appl. Phys. Lett. 52, 1249 (1988).

[24] Q.D. Qian, J. Qiu, M.R. Melloch, J.A. Cooper, L.A. Kolodziejski, R.L. Gunshor, Appl. Phys. Lett. 54, 1359 (1989).

[25] J. Qiu, Q.D. Qian, R.L. Gunshor, M. Kobayashi, D.R. Menke, D. Li, N. Otsuka, Appl. Phys. Lett. 56, 1272 (1990).

[26] M.L. Terman, Solid-State Electron. 5, 285 (1962).

[27] Sung-koo Chung, Y. Wu, K.L. Wang, N.H. Sheng, C.P. Lee, , D.L. Miller, IEEE Trans. Electron Devices ED-34, 149 (1987).

[28] J. Tersoff, Phys. Rev. Lett. 56, 2755 (1986). 
[29] K.J. Mackey, P.M.G. Allen, W.G. Herrenden-Harker, R.H. Williams, C.R. Whitehouse, G.M. Williams, Appl. Phys. Lett. 49, 354 (1986).

[30] R.G. van Welzenis, B.K. Ridley, Solid-State Electron. 27, 113 (1984).

[31] L.A. Kolodziejski, R.L. Gunshor, N. Otsuka, A.V. Nurmikko, Mat. Res. Soc. Symp. 102, 113 (1988).

[32] J.L. Glenn, O. Sungki, L.A. Kolodziejski, R.L. Gunshor, M. Kobayashi, D. Li, N. Otsuka J. Vac. Sci. Technol. 7, 249 (1989). 